\section{One-year outcome after intravitreal ranibizumab for large, serous pigment epithelial detachment secondary to age-related macular degeneration}

\begin{abstract}
Aim To report the effects of intravitreal ranibizumab therapy for large, serous pigment epithelial detachment (PED), secondary to age-related macular degeneration, and occupying more than $50 \%$ of the total lesion area.

Materials and methods In a retrospective case series, visual acuity, ocular coherence tomography (OCT), and safety data were collected for 19 eyes of 19 patients, with serous PED and evidence of disease progression. Intravitreal ranibizumab of $0.5 \mathrm{mg}$ was given with a loading phase of three consecutive monthly injections, followed by monthly
\end{abstract}

Eye Department, Eye Clinic, St James's University Hospital, Leeds, UK

Correspondence: S Arora, 9, Alma Close, Kirkella, East Yorkshire HU107LH, UK Tel: + 44 (0) 7973816129 ; Fax: + 44 (0) 1482605327 E-mail: seemaarora@ doctors.org.uk

Received: 22 July 2010 Accepted in revised form: 29 March 2011

Published online: 20 May 2011

This work was presented at the North of England Ophthalmological Society Spring Meeting in

Manchester in March 2010
S Arora and M McKibbin

are comparable to those seen in multicentre, phase 3 trials of other lesion types, and were obtained without the need for either monthly, fixed treatment, or for continued treatment until the PED resolves.

Eye (2011) 25, 1034-1038; doi:10.1038/eye.2011.115; published online 20 May 2011

Keywords: pigment epithelial detachment; age-related macular degeneration; ranibizumab

\section{Introduction}

Serous pigment epithelial detachments (PED) are rare, but important features of neovascular age-related macular degeneration (AMD), leading to visual loss through the accumulation of fluid under the retinal pigment epithelium (RPE) and neurosensory retina, and secondary complications including atrophy of the outer retina and a tear or rip of the RPE. At present, there is little good evidence to inform the management of large, serous PED, which involve more than $50 \%$ of the total lesion area. These large serous PED have traditionally been excluded from phase 3 clinical trials, including TAP, ANCHOR, and MARINA. ${ }^{1-3}$ Therefore, the results of these trials cannot be extrapolated to the management of these lesions. This retrospective case series reports the safety and efficacy of intravitreal ranibizumab (Lucentis, Genentech, South San Francisco, CA, USA) monotherapy as a treatment for large serous PED secondary to AMD. 


\section{Methods}

In a retrospective, interventional case series, baseline and follow-up data were collected for eyes with large serous PED, occupying more than $50 \%$ of the total lesion area, and treated with $0.5 \mathrm{mg}$ of intravitreal ranibizumab monotherapy. This data included best-corrected ETDRS letter score, maximum PED height on ocular coherence tomography (OCT), the presence of cysts or sub-retinal fluid on OCT, and the presence of relevant ocular complications. All patients were scanned with Stratus OCT (Carl Zeiss, Germany) using the macular thickness protocol, and/or $6 \mathrm{~mm}$ horizontal and vertical line scans. The PED height was measured manually on the OCT monitor using the built-in manual calliper tool. The indication to start treatment was disease progression, characterised by one or more of the following: decreasing visual acuity, new sub-retinal haemorrhage, or increasing evidence of disease activity on OCT. Before treatment, fluorescein angiography was performed to determine the baseline lesion characteristics, and the images were evaluated by a single observer. In accordance with published criteria, a serous PED was characterised by a smooth elevation of the RPE with early, uniform hyperfluorescence on angiography that remains bright, and with sharp borders during the later phases of the angiogram. ${ }^{4}$ According to baseline fluorescein angiography and OCT examination findings, serous PED were classified into two subgroups, namely clinically avascular serous PED and vascularised or CNVassociated serous PED (Figure 1). Lesions were felt to be vascularised when there was evidence of any of the following: sub-retinal haemorrhage, speckled hyperfluorescence on fluorescein angiography, consistent with occult $\mathrm{CNV}$, sub-retinal fluid, or intra-retinal cysts on OCT examination. Lack of these features in the presence of smoothly elevated dome-shaped PED defined avascular serous PED. The objective of classifying serous PED into two groups was to see if vascularised serous PED with intra or subretinal fluid shows better functional response to intravitreal ranibizumab than avascular serous PED (retinal angiomatous proliferations and fibro-vascular PEDs without a large serous PED were excluded from the analysis).

In accordance with the European marketing authorisation, treatment was given as an initial loading phase of three consecutive monthly injections, followed by a maintenance phase of repeated injection as required, according to visual acuity, OCT, and clinical examination
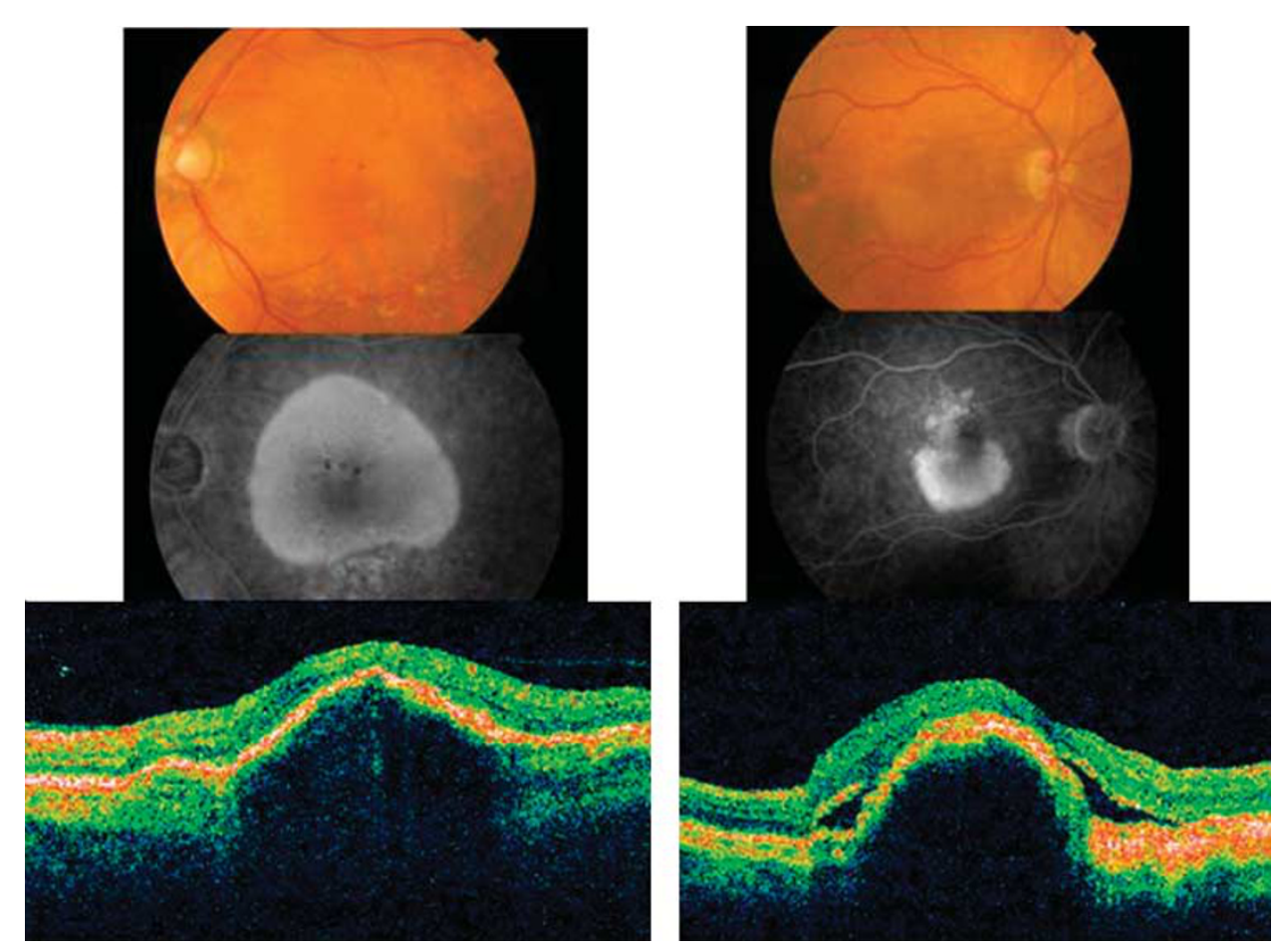

Figure 1 A large serous PED is seen of the colour fundus photograph. There is increasing hyperfluorescence on fluorescein angiography, but no leakage. Blocked fluorescence corresponds to pigment seen on colour photography. A serous PED, without sub-retinal fluid or intra-retinal cysts, is seen on OCT examination. This lesion was felt to be avascular. A serous PED is seen below the fovea on colour and fluorescein angiography. There is speckled hyperfluorescence on angiography above the PED, consistent with occult CNV. There is sub-retinal and sub-RPE fluid on OCT examination. This lesion was felt to be vascularised. 
findings. New or persistent sub-retinal fluid, retinal cysts, or thickening were considered to be indications for retreatment, but not the presence of serous PED alone.

Statistical analysis was performed using the Wilcoxon Matched-Pairs Signed Rank, Wilcoxon Two Sample and correlation tests as appropriate, with an online statistical program (http:/ / www.fon.hum.uva.nl/Service/ Statistics.html).

\section{Results}

A total of 20 eyes of 20 Caucasian patients (13 females, average age 76.5 years) were identified, and 19 eyes completed 12 months of follow-up and were included in the analysis. Baseline characteristics are shown in Table 1. For the entire series, 18/19 (95\%) eyes avoided moderate visual loss at the 12-month examination, 12 eyes (63\%) had an increase in ETDRS letter score from baseline, and five eyes $(26 \%)$ had a gain of 15 or more letters. For the 12 eyes with a letter score gain, five $(42 \%)$ had the maximum letter score recorded after the initial loading phase of three injections, and seven (58\%) patients had largest gain at the month-12 visit. The mean number of injections before the 12-month visit was 7.4.

The mean ETDRS letter score at baseline was 61 letters for the nine avascular PED, and 52 letters for the 10 vascularised PED. At month 12, the mean letter score change was a gain of six and four letters for the avascular and vascularised PED, respectively (Table 2). No significant differences in visual acuity outcomes were seen between the subgroups at baseline $(P=0.098)$, and at months $3(P=0.37), 6(P=0.59)$, and $12(P=0.34)$.

Table 1 Patient demographics and baseline characteristics

\begin{tabular}{lcc}
\hline Characteristics & Avascular-9 & Vascularised-10 \\
\hline $\begin{array}{l}\text { Gender } \\
\text { Male }\end{array}$ & 3 & 3 \\
$\quad$ Female & 6 & 7 \\
Mean age (years) & 79 & 74 \\
Baseline vision & & \\
$\quad$ Mean letter score & 61 & 52 \\
Range & $46-73$ & $36-70$ \\
Baseline PED characteristics & & \\
$\quad$ Mean total area (sqmm) & 4.1 & 5.9 \\
Range (sqmm) & $1.54-11.8$ & $2.3-8.8$ \\
$\quad$ Mean percentage of PED & & $80.60 \%$ \\
Range & & $59.1-100 \%{ }^{\mathrm{a}}$ \\
Average injections & 5.4 & 8.4 \\
\hline
\end{tabular}

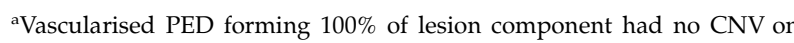
angiography, but had SRF and intraretinal cysts on OCT.
There was also no difference in relation to the percentage with $\leq 15$ letter loss, $\geq 1$ letter gain, and $\geq 15$ letter gain (Figure 2).

The mean PED height in the vascularised PED subgroup was significantly larger than in avascular PED sub-group at all study intervals (Table 3). The mean (SD) change in PED height from baseline to 12 months was $-64.11 \mu \mathrm{m}( \pm 87.34)$ for the avascular PED group, and $-80 \mu \mathrm{m}$ ( \pm 50.16$)$ for vascularised PED group (Table 3 ). The mean decrease in PED height from baseline to 12 months was statistically significant for both the avascular PED group $(P=0.05)$ and the vascularised PED group $(P=0.0019)$, but the difference between the two groups was not significant. In none of the patients was the PED noted to have disappeared completely during the follow-up period. The study did not find any correlation between the baseline PED height and baseline visual acuity ( $r=-0.0263, P$-value 0.91$)$, or change in the PED height and change in the letter score $(r=0.3, P$-value 0.17$)$, or baseline PED height and change in PED height ( $r=-0.35, P$-value 0.13$)$.

Treatment was well tolerated, and there were no recorded cases of presumed infectious endophthalmitis. One eye was noted to have had an RPE rip during the

Table 2 Mean ETDRS letter scores

\begin{tabular}{lcccc}
\hline PED & Baseline & 3 months & 6 months & 12 months \\
\hline Avascular & 61 & 64 & 62 & 67 \\
SD & \pm 9.5 & \pm 9.7 & \pm 10.88 & \pm 11.21 \\
95\% CI & $53.2-67.86$ & $56.08-71.03$ & $55.38-69.5$ & $60.12-74.76$ \\
& & & & \\
Vascularised & 52 & 55 & 57 & 56 \\
$\quad$ SD & \pm 11.53 & \pm 16.13 & \pm 16.8 & \pm 21.05 \\
95\% CI & $44.55-58.85$ & $44.9-65.21$ & $46.89-67.71$ & $42.95-69.05$ \\
P-value & 0.094 & 0.37 & 0.59 & 0.34 \\
\hline
\end{tabular}

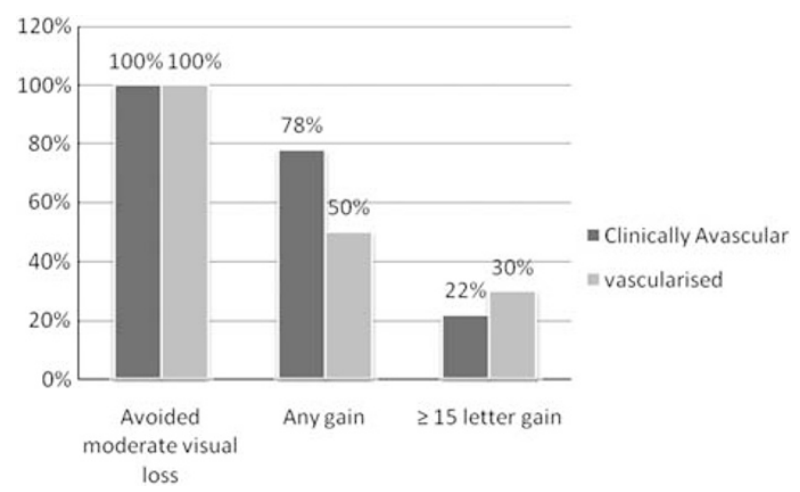

Figure 2 Percentage of patients with letter score changes at month 12 in the avascular and vascularised PED subgroups. Moderate visual loss is defined as loss of 15 or more letters. 
Table 3 Mean PED height $( \pm S D)$

\begin{tabular}{lcccc}
\hline Patient & Baseline & 3 months & 6 months & 12 months \\
\hline Avascular $(\mu \mathrm{m})$ & $292( \pm 103)$ & $286( \pm 85.7)$ & $257( \pm 84.9)$ & $228( \pm 95.5)$ \\
Vascularised $(\mu \mathrm{m})$ & $498( \pm 168)$ & $445( \pm 138)$ & $440( \pm 121)$ & $418( \pm 160)$ \\
$P$-value & 0.01 & 0.017 & 0.004 & 0.01 \\
\hline
\end{tabular}

follow-up period. This complication occurred after the fifth intravitreal injection. This patient received one further intravitreal injection, but developed a large subretinal haemorrhage and, despite a pars plana vitrectomy to remove the sub-retinal haemorrhage, the visual acuity change after 12 months was a decrease of 23 letters.

\section{Discussion}

The findings of this study are similar to those in the ANCHOR and MARINA studies in that, after 1 year, 95\% patients avoided moderate visual loss (15 or more letters), and $25 \%$ had a gain of 15 or more ETDRS letters. However, in the ANCHOR and MARINA studies, the maximum visual gain was often seen during the first 3 months of treatment. In contrast, in this study, more than half of the eyes with gain in ETDRS letter score had their maximum gain at the end of 1 year, suggesting that treatment response in lesions with large serous PED may be slower. This finding would also suggest that treatment might have to be continued for a longer period to obtain the maximum visual acuity benefit.

For the purpose of this study, we classified serous PED in to avascular and vascularised serous PED. We expected the vascularised serous PED to show better response due to presence of intra-retinal oedema and sub-retinal fluid, as these features can effectively be reduced by anti-VEGF therapy. However, this study did not show any notable difference in the visual acuity outcomes between the serous avascular and vascularised PED after 3, 6, and 12 months of follow-up. This suggests that, when associated with disease progression, the differentiation into avascular and vascularised serous PED may be mistaken.

In this study, although there was a trend towards a decrease in PED height during the follow-up, at no point was the PED noted to have resolved completely. The change in the PED height was more in vascularised group, but this change did not have any significant correlation with higher mean-baseline PED height of vascularised PED. The patients still had improvement in visual acuity, even without complete resolution of PED in both the groups. This suggests that complete resolution of PED is not an essential morphological effect to appreciate visual benefits and, in our opinion, retreatment should not be guided by this morphological change. In $60 \%$ of eyes with vascularised PED, complete resolution of morphological features such as IRF/cysts and SRF were accompanied by improvement in visual acuity, and it appears that this anatomical change may be more relevant for improving visual function, and thus for retreatment.

The findings of our study are consistent with that of Ritter et al ${ }^{5}$ (7-avascular, 5-drusenoid PED) in terms of the visual acuity response and irrelevance of change in the morphology of PED for stabilisation of visual outcome. Ritter et $a l^{5}$ treated their patients with three or six fixed injections. They noted stabilisation of vision in all cases and a decrease in PED volume of $42 \%$ at 6 months, but this morphological effect was temporary and did not last at 9 and 12 months.

Lommatzsch et $a l^{6}$ reported superior visual acuity and functional outcomes with ranibizumab and bevacizumab therapy, in comparison with either pegaptanib therapy alone, or a combination of photodynamic therapy and intravitreal triamcinolone in serous PED, with CNV and RAP lesions. That study did not include avascular PED and the mean follow-up was of 24 weeks. Overall, the best-corrected visual acuity of $0.78 \log$ MAR before treatment could be improved by about $0.066 \log$ MAR after treatment. Mean retinal thickness decreased in all patients with PED, by about $64.06 \mu \mathrm{m}$, and the mean value of the manually calculated height decreased by about 0.98 units.

A challenging aspect of managing eyes with large serous PED is the risk of complications including both RPE rip and/or sub-retinal haemorrhage. In the studies by Ritter et $a l^{5}$ and Lommatzsch et $a l^{6}{ }^{6}$ the incidence of RPE rip varied from 8 to $12 \%$, respectively. Chiang et $a l^{7}$ have reported large PED basal diameter and vertical height of PED to be the predictors of increased risk of RPE rip (17\%) in eyes (60 eyes) treated with anti VEGF (ranibizumab, bevacizumab, and pegaptanib). In this study, in RPE tear group, the median greatest linear diameter on FFA was $3.2 \mathrm{~mm}$ and median maximum PED height on OCT was $394 \mu \mathrm{m}$. In our study, the median basal diameter was slightly lower $(2.9 \mathrm{~mm})$ and median maximum PED height was higher $(472 \mu \mathrm{m})$ than that reported in the study by Chiang et al. ${ }^{7}$ A single eye that developed RPE tear in our study had vertical PED height of $634 \mu \mathrm{m}$. The mechanism for formation of RPE rip 
following anti-angiogenic treatment has been suggested to be either spontaneous rupture or increasing contraction of CNV due to anti-angiogenic effect. ${ }^{7}$

The limitations of this study include the small sample size and retrospective data collection. Indocyanine angiography was not routinely performed to confirm the presence of $\mathrm{CNV}$, and there was no observerindependent algorithm for measurement of PED height. In addition, treatment was given with a loading phase of three fixed, monthly injections, followed by a maintenance phase of repeated injection according to disease activity. Therefore, the outcomes in this study may be different to those with fixed, monthly treatment. However, large serous PED, occupying more than $50 \%$ of the total lesion area are rare and the findings of our retrospective series are in keeping with those of others.

In conclusion, intra-vitreal ranibizumab was found to be a safe and effective therapy for the treatment of large serous PED associated with progression. Stable acuity at 1 year was seen in $95 \%$ of eyes, and $25 \%$ had a gain of at least 15 ETDRS letters. These outcomes were obtained without the need for monthly, fixed treatment, and repeat treatment was not prompted by persistence of the PED alone.

\section{Summary}

What was known before

- The best management of large, serous pigment epithelial detachments secondary to AMD, occupying more than $50 \%$ of the total lesion area and associated with disease progression, is unclear. These lesions have traditionally been excluded from multi-centre phase 3 clinical trials.

What this study adds

- This study reports that intravitreal ranibizumab monotherpy is safe and effective treatment for large, serous PED. Stable and improved acuity was recorded in 95 and $63 \%$ of subjects using a loading phase of three fixed, monthly injections, followed by further injection as required. Complete resolution of the PED was not seen in any case at the month-12 examination.

\section{Conflict of interest}

The authors declare no conflict of interest.

\section{Acknowledgements}

We thank Mr Arthur Kang'ombe, and Dr Catherine E Hewitt (Department of Health Sciences, University of York, York) for their advice on statistics.

\section{References}

1 Photodynamic therapy of subfoveal choroidal neovascularisation in age-related macular degeneration with verteporfin: one-year results of 2 randomized clinical trialsTAP report. Treatment of age-related macular degeneration with photodynamic therapy (TAP) Study Group. Arch Ophthalmol 1999; 117: 1329-1345.

2 Rosenfeld PJ, Brown DM, Heier JS, Boyer DS, Kaiser PK, Chung $\mathrm{CY}$ et al. Ranibizumab for neovascular age related macular degeneration. N Engl J Med 2006; 355: 1419-1431.

3 Brown DM, Kaiser PK, Michels M, Soubrane G, Heier JS, Kim RY et al. Ranibizumab versus verteporfin for neovascular age-related macular degeneration. $N$ Engl J Med 2006; 355: 1432-1444.

4 Barbazetto I, Burdan A, Bressler NM, Bressler SB, Haynes L, Kapetanios AD et al., Treatment of Age-Related Macular Degeneration with Photodynamic Therapy Study Group; Verteporfin in Photodynamic Therapy Study Group. Photodynamic therapy of subfoveal choroidal neovascularisation with verteporfin: fluorescein angiographic guidelines for evaluation and treatment-TAP and VIP report No. 2. Arch Ophthalmol 2003; 121: 1253-1268.

5 Ritter M, Bolz M, Sacu S, Dea'k G, Kiss C, Pruente C et al. Effect of intravitreal ranibizumab in avascular pigment epithelial detachment. Eye (Lond) 2009; 24(6): 962-968.

6 Lommatzsch A, Heimes B, Gutfleisch M, Spital G, Zeimer M, Pauleikhoff D. Serous pigment epithelial detachment in age-related macular degeneration: comparison of different treatments. Eye 2009; 23: 2163-2168.

7 Chiang A, Chang LK, Yu F, Sarraf D. Predictors of anti-VEGF associated retinal pigment epithelial tear using FA and OCT analysis. Retina 2008; 28: 1265-1269. 